\title{
Ferulizaciones diente-implante
}

\author{
HERNÁNDEZ G* \\ FERRÚS J** \\ BASCONES A***
}

Hernández G, Ferrús J, Bascones A. Ferulizaciones dienteimplante. Av Periodon Implantol. 2005; 17, 3: 165-174.

\begin{abstract}
RESUIMEN
Se presenta una revisión sobre la posibilidad terapéutica del empleo de la ferulización entre dientes e implantes. Tras una breve introducción, se establece una serie de diferencias biomecánicas entre dos estructuras tan dispares como son el diente y el implante. Posteriormente, se exponen una serie de puntos que los distintos autores han achacado a este sistema protésico para luego describir los distintos diseños que se pueden aplicar (rígidos y semirrígidos). A continuación, se efectúa una revisión bibliográfica acerca de la evolución a corto y largo plazo de estos sistemas. Por último, se elabora una relación de ventajas, desventajas e indicaciones de la ferulización diente-implante, para terminar estableciendo unas conclusiones.
\end{abstract}

\section{PALABRAS CLAVE}

Conexión Diente-implante, prótesis implantodentosoportada, ferulización.

Aceptado para publicación: Septiembre 2005.

\section{INTRODUCCIÓN}

El tratamiento implantológico es, como se ha venido demostrando desde hace bastante tiempo, bastante previsible y alentador, y en la actualidad, las posibilidades técnicas que los nuevos sistemas de implantes pueden ofrecer hacen que los profesionales se planteen nuevos retos. En muchos casos, la rehabilitación del sistema estomatognático se lleva a cabo mediante prótesis implantosoportadas convencionales, pero llama la atención el hecho de que casi nunca se plantea la posibilidad de unir protésicamente dos estructuras tan diferentes como son diente e implante, quizá por falta de evidencias científicas que permitan abogar por ella.

\section{CONSIDERACIONES BIOIMECÁNICAS}

Después del éxito que los implantes han ido cose- chando en la literatura, y el entusiasmo de algunos profesionales (unido algunas veces al potente marketing de algunas casas comerciales), la tendencia actual hace que el paciente y muchas veces el profesional odontólogo vea en el implante un sustituto ideal del diente, y esto no es correcto, teniendo en cuenta las grandes diferencias que presentan uno y otro, diferencias que hacen que el implante presente un comportamiento biomecánico que no puede compararse al regulado equilibrio funcional que ostenta un diente integrado en el sistema estomatognático, y condicionan que las estructuras protésicas que adaptamos a uno deban ser diferentes a las del otro.

En primer lugar, se debe resaltar el hecho de que el diente consta de un elemento amortiguador que es el ligamento periodontal. Este componente le confiere un carácter resilente ante las fuerzas oclusales, de modo que puede compensar el estrés oclusal mediante pequeños movimientos que se han cifrado entre las 200 y las 300 micras ante fuerzas masticatorias fisioló-

* Odontólogo. Alumno de Curso de Experto en Clínica Periodontal. Facultad de Odontología UCM.

** Alumno del Master de Periodoncia. Facultad de Odontologia UCM.

*** Catedrático de Medicina Bucal y Periodoncia. Facultad de Odontología UCM. 
gicas. El implante, por el contrario, carece de ligamento periodontal. Se habla de una serie de fibras que, durante la fase de osteointegración, rodean al implante y se insertan en el hueso, un "pseudoperiodonto", y en algún momento se llegó a pensar que los implantes presentaban una cierta movilidad merced a estos fenómenos, pero finalmente se ha visto que dichas fibras no pueden considerarse ni mucho menos como un sustituto del ligamento periodontal y, en consecuencia, las fuerzas originadas por el implante ante el hueso sólo pueden considerarse de compresión y cizallamiento.

La resistencia de los tejidos de soporte a la aplicación de fuerzas externas empieza a aumentar cuando el diente inicia su movimiento de rotación, rotación que es detenida cuando el par de resistencia iguala el momento producido por la fuerza externa. El movimiento, como resultado de momentos descompensados, es controlado y reducido mediante cambios en las variables de la ecuación. La magnitud de las fuerzas debe ser considerada constante. Teniendo en cuenta que la máxima fuerza aplicable al sistema depende de la capacidad muscular y el umbral de dolor del individuo, podemos pensar, para aumentar la estabilidad del sistema, en:

- Reducir el brazo de palanca (acortando el diente, por ejemplo).

- Aumentar la resistencia dentro del periodonto mediante la ferulización a los dientes adyacentes, o a otras estructuras como los implantes.

\section{RIESGOS A TENER EN CUENTA EN LAS PRÓTESIS IMPLANTODENTOSOPORTADAS:}

En primer lugar, desde que se pensó en la posibilidad de ferulizar dientes e implantes y se elaboraron los primeros ensayos y estudios experimentales, ya se pensó en el riesgo, dada la distinta naturaleza de las dos estructuras pilares, de una intrusión del diente pilar. Los autores pensaban que la carga de las fuerzas oclusales sobre la prótesis implantodentosoportada producían un movimiento de balanceo en el que el diente se intruía en su movimiento natural y que, en una serie continua de intrusiones y extrusiones, finalmente, los movimientos de intrusión no se corregirían y el diente pilar, a largo plazo, acabaría en una posición más apical y anquilosándose.

Del mismo modo, este fenómeno de palanca debido a la diferente naturaleza de ambas estructuras era entendido por los diversos autores como una sobrecarga de la estructura protésica, que se vería así constantemente sometida a cargas anómalas que podrían provocar fracturas protésicas o problemas en el ajuste de la prótesis en el pilar natural.

En estudios posteriores, se vio que la aplicación de este tipo de prótesis, al ferulizar diente e implante de forma rígida, producía un desplazamiento de la carga hacia el implante pilar, sobrecargándolo y disminuyendo su vida útil, con riesgo de reabsorción ósea y de fractura de los distintos elementos implantarios.

\section{POSIBILIDADES DE DISEÑO}

\section{INTRODUCCIÓN}

En un principio, y debido a las observaciones realizadas anteriormente, estas ferulizaciones se realizaban mediante un sistema de ataches resilientes, aduciendo una mejor distribución de las cargas, pero la evolución del conocimiento implantológico y protésico ha llevado a replantear estos sistemas en beneficio de otros, al observar fenómenos de migración dentaria. Con el tiempo se vio, mediante distintos estudios, que la aplicación de estas conexiones semirrígidas no presentaban ninguna justificación, favoreciendo finalmente a las conexiones rígidas, como han visto Cavicchia y Bravi (1999) y otros estudios. Muchos estudios han visto que estas conexiones rígidas funcionan sin complicaciones, como veremos posteriormente, y se ha encontrado que la elasticidad del titanio y de los tornillos de fijación es suficiente para compensar el movimiento dental, pero también existen diseños basados en conexiones resilientes que funcionan según estudios bastante actuales.

Por todo ello parece que los diseños pueden agruparse en dos grandes familias:

\section{CONEXIÓN SEMIRRÍGIDA:}

Muchos autores proponen que una solución al problema de la unión de los dientes y los implantes, dada su diferente naturaleza, se efectúe mediante conectores semirrígidos, que rompan las fuerzas alegando que, al individualizar los sectores de la prótesis, cada pilar soporta la carga funcional de forma óptima, en contra de la ferulización rígida en la que aparecerían fenómenos de intrusión dental y sobrecargas del implante. Existen diversas posibilidades dentro de los conectores semirrígidos que se han ido exponiendo en la literatura y que se pueden agrupar básicamente en dos grupos de ruptores de fuerzas: 
- Componente hembra en dientes y macho en
implante.

- Componente macho en dientes y hembra en implante.

La literatura ha visto que, de estos dos diseños, el más recomendable es el segundo, pero que, en comparación con los sistemas rígidos, parece que, en general, las conexiones semirrígidas no siempre son fiables, sino que, paradójicamente, son estos sistemas los que más relación parecen tener con la aparición de fenómenos intrusivos y además que, al suponer una complicación del diseño protésico, se suela optar por los conectores rígidos, que parecen no presentar mayores complicaciones.

\section{CONEXIÓN RÍGIDA}

Consiste en la ferulización diente implante sin establecer ningún elemento que rompa las fuerzas generadas en la prótesis.

Existe mucha controversia acerca de la idoneidad de esta conexión en contra de la no rígida. Si bien esta última presenta problemas en cuanto a la posibilidad de intrusión del diente en cualquiera de sus posibilidades de diseño, algunos estudios a largo plazo ven que en este sistema se sobrecarga más el implante y se pierde más hueso (Naert et al., 2001), mientras que otros no ven esta relación. El estudio de Naert et al. es prospectivo a 15 años, y en él se puede observar que, aplicando en el análisis distintas variables se llega a la conclusión de que la ferulización rígida presenta una mayor cuantía de reaborción ósea. Si no tuviéramos en cuenta estas y otras variables en el análisis, el resultado hubiera redundado en una menor diferencia entre las prótesis implantosoportadas y las prótesis implantodentosoportadas rígidas, con lo que tendríamos un resultado muy similar al que encontraron Gunne et al (1999) o Hosny et al. (2000).

Nishimura et al. (1999), mediante el empleo de análisis fotoelástico, observaron una considerable reducción del estrés aplicado sobre el implante al establecer conexiones semirrígidas. En este estudio se ve cómo las aplicaciones de cargas oclusales sobre el diente aumentaban el estrés apical y crestalmente al implante conectado rígidamente. En una conexión semirrígida se veía cómo el conector disminuía la transferencia de carga en dirección hembra-macho. Dicho conector se orientaba con el fin acomodar la movilidad del diente pilar y así reducir el estrés de cantilever desde el diente conectado al pilar implantario.

Wang et al. (1999) diseñó un sistema robot para producir movimientos mandibulares simulados y fuerzas oclusales de contacto, encontrando altos momentos de flexión en implantes conectados a dientes de forma rígida.

Tanto el estudio de Naert et al como otros (Van Oosterwyck et al., 1998) ven que la cuantía de pérdida ósea anual es el triple que en prótesis implantosoportadas, aunque a los seis meses la situación se normaliza y la pérdida anual es similar a la ocurrida en conexiones semirrígidas.

Esta situación nos plantea un dilema: si bien está bastante claro que el empleo de conectores semirrígidos induce la aparición de fenómenos de intrusión del diente natural, podemos plantearnos la conexión rígida, pero esta provoca una mayor (aunque controlada tras la fase inicial) reabsorción ósea, con lo que el profesional se debate entre dos posibilidades entre las que tiene que escoger la de menor riesgo pero, a la vez, mayor aceptación por parte del paciente. A este respecto, tendría más sentido decantarse por la ferulización rígida pues la reabsorción ósea, que se va a controlar favorablemente en el período secundario a la fase de remodelación, no es visible por el paciente, que sí puede apreciar la intrusión que, además, conlleva en algunos casos importantes problemas al modificar la oclusión del paciente. Además, el hecho de establecer un atache semirrígido, como se ha apuntado anteriormente, complica el diseño de la prótesis y puede afectar a la oclusión.

Sin embargo, cabe pensar en intentar dar solución a este problema de la sobrecarga del implante en conexiones rígidas, y en este sentido se han propuesto diversas posibilidades:

\section{- Elementos implantarios resilientes}

Dada la capacidad que presenta el diente natural para amortiguar mediante pequeños movimientos el estrés ocasionado por las fuerzas oclusales, cabe la posibilidad de hacer que el implante intente emular esta resiliencia mediante algún aditamento especial que haga las veces de ligamento periodontal. Un tipo de implante con estas características sería el fabricado por la casa IMZ. El sistema fue ideado por el doctor Axel Kirsch y consiste en el uso de polioximetileno, un material resiliente que disipe las fuerzas originadas sobre la cabeza del implante. El polioximetileno, al ser biocompatible y presentar una alta resistencia al creep, parecía muy indicado para soportar el estrés especial al que se sometían los implantes en algunos casos. Se supone que estos pilares especiales actuarían en el implante reduciendo el estrés producido pero, en lo que se refiere a las prótesis implantosoportadas convencionales, la literatura ha observado que el sistema no resulta eficaz, y la estructura protésica aca- 
baba sobrecargándose y fracturándose a medio y largo plazo. Sin embargo, algunos autores apuntan la posibilidad del empleo de estos sistemas si adicionamos su efecto amortiguador al hecho de que el implante pueda estar ferulizado a un diente, con lo que la distribución de las fuerzas sería más amortiguada. Así lo vieron Cavicchia y Bravi (1999). Sin embargo, existen otros estudios que no ven la utilidad de estos sistemas en ninguna de las dos posibilidades. McGlumphy, Campagni y Peterson (1988), realizaron un estudio fotoelástico en el que no vieron diferencias estadísticamente significativas entre las cargas transmitidas por implantes con sistemas resilientes y las transmitidas por implantes convencionales. También Brunski (1988) vio que, en una ferulización dienteimplante, el $70-80 \%$ de la carga era soportada por el implante mientras que un $20-30 \%$ era soportada por el diente natural, situación que cambiaba de forma poco notoria al aplicar elementos intramóviles.

\section{- Caras oclusales de materiales resilientes}

En este sentido la literatura tiene muy pocos estudios realizados. Se ha pensado en el empleo de dientes de resina por su capacidad de absorción de cargas pero ya es conocida la menor estética de estos materiales en comparación con las nuevas porcelanas, su rugosidad, que la hace subsidiaria del acúmulo de placa bacteriana y el riesgo de fractura del material, con lo que no se piensa en esta opción como posible.

\section{- Eliminación intencionada de contactos oclusales}

Con el fin de disminuir la sobrecarga del implante, se plantea la disminución de su papel en la oclusión, reduciendo intencionadamente los contactos oclusales con los dientes antagonistas. Richter (1989) realizó un estudio en el que evaluó el efecto de esta posibilidad, viendo que una disminución de 15 micras en la oclusión fisiológica del implante reducía drásticamente la sobrecarga del implante. Se trataría de una posibilidad a tener en cuenta ante tramos cortos de restauración, en los que la menor oclusión del implante no supondría una sobrecarga excesiva del diente natural, pero no en tramos edéntulos largos o bien en casos en los que se ferulizan varios implantes a un solo diente.

\section{- Empleo de implantes Branemark}

Rangert et al., (1991), han visto que la resiliencia natural de los implantes Bränemark convencionales podría suponer una solución más al problema de la sobrecarga de los implantes, disipando el estrés oclusal originado sobre la prótesis implantodentosoporta- da, si bien debemos tener en cuenta que la evolución en los sistemas de implantes es debida a que los nuevos sistemas presentan mejores resultados a largo plazo y que el empleo del sistema Bränemark convencional, con implantes mecanizados de superficies lisas no parece en la actualidad una indicación.

\section{- Ferulización de un implante a un mayor número de dientes}

Como parece ser que añadir aditamentos resilientes a los implantes con el fin de reducir el estrés oclusal generado no es una técnica muy fiable, como se ha visto e la literatura, se puede pensar a la inversa, es decir, hacer que el sector protésico dentosoportado sea más rígido, más similar al implante. Esto se podría llevar a cabo mediante la ferulización de más dientes al mismo implante. Naert et al. así lo han visto, observando una menor movilidad unitaria de los dientes, más comparable a la de los implantes. Sin embargo, es preciso tener en cuenta que esta solución requeriría el tallado de más dientes, siendo menos conservadores, entrando por tanto en la contraindicación más que en la justificación.

\section{ÉXITO DE LA FERULIZACIÓN CON EL TIEIMPO}

\section{INTRODUCCIÓN}

En lo que se refiere al tema que estamos tratando es preciso destacar que, en primer lugar y, desafortunadamente, existen pocos estudios a largo plazo y, que, además, los criterios de éxito o fracaso, como en otros campos de la Odontología, no está estandarizados, por lo que los resultados pueden variar mucho de unos estudios a otros en función del material y métodos de los investigadores. En la mayoría de los estudios, por ejemplo, el principal criterio de éxito era la simple supervivencia del implante, y no se tenían en cuenta otras posibles complicaciones, ni se evaluaban otras variables importantes en el éxito de la prótesis como es la pérdida marginal de hueso. En estos casos, los resultados obtenidos reflejan la evolución de las prótesis implantodentosoportadas en condiciones poco definidas y en líneas muy generales. Un ejemplo de qué parámetros se pueden considerar en el seguimiento de una prótesis implantodentosoportadas para evaluar el éxito podría ser el propuesto por Olsson et al (1995), que estudia:

- Supervivencia del implante.

- Estabilidad del puente.

- Movilidad dentaria. 
- Nivel de hueso marginal mediante radiografía.

- Reacciones de los tejidos blandos marginales:

- Acúmulo de placa bacteriana.

- Gingivitis.

- Profundidad de bolsa.

- Nivel de anclaje.

- Alteraciones sensoriales de mejilla y labio.

- Complicaciones técnicas.

La mayoría de estos parámetros son subjetivos, por lo que es muy necesaria la estandarización de criterios para todos los observadores que entren en el estudio.

En cuanto a la planificación, también existen muchas variables como son el número de implantes, la longitud de la prótesis, el diseño protésico... (variables de confusión), que influyen de manera decisiva en la evolución de la prótesis a largo plazo, y que tampoco se suelen tener en cuenta en muchas ocasiones, sino que sólo se considera el conjunto de la ferulizaciones diente-implante sin entrar en mayores disquisiciones acerca del tipo de conector o la distribución y número de implantes, con lo que las conclusiones se encuentran aplicadas a un conjunto muy global con elementos de distintas características y diferentes entre sí.

Además, se puede apreciar en muchos artículos el hecho de que los dientes pilares empleados en estos estudios tenían en la mayoría de los casos buen soporte óseo y movilidad mínima, con lo que las conclusiones obtenidas a este respecto no podrían aplicarse a todos los casos con fines asistenciales. Sería relevante resaltar la importancia de tener en cuenta todas las variables, como sugiere Hutton et al. (1995)

Sin embargo, y a pesar de la necesidad de más estudios a largo plazo y teniendo en cuenta el mayor número de variables posible, se pueden extrapolar una serie de conclusiones generales que nos permitan enfocar los distintos casos que se presentan al profesional hacia uno $u$ otro plan de tratamiento.

En resumen, si anteriormente la tendencia común iba dirigida a considerar estas restauraciones como fiables y exitosas sólo a corto plazo, y que, si bien en los primeros años existe una tasa de supervivencia considerable, en la evolución de la distribución de cargas finalmente se producen alteraciones y migraciones dentarias que suponen el fracaso de la prótesis, los resultados obtenidos en distintos estudios hacen pensar en lo contrario. Se tiende a pensar en este tipo de prótesis como una práctica sólo a efectuar en casos extremos sin plantearse su posibilidad como alternativa a un tratamiento más agresivo o costoso, pero la evidencia científica arroja datos que hacen pensar en lo contrario, y hay estudios, no sólo a corto sino a largo plazo en los que las tasas de supervivencia igualan (o incluso superan) a los resultados obtenidos con las restauraciones implantosoportadas.

\section{EVOLUCIÓN A CORTO PLAZO}

Brägger et al (2001) han visto en un estudio comparativo con prótesis parciales fijas sobre dientes y prótesis parciales fijas sobre implantes, que las prótesis parciales fijas sobre dientes ferulizados a implantes no presentan una evidencia significativa de complicaciones técnicas o biológicas, en un estudio realizado tras 4-5 años de función. Vieron que, a los 5 años, la supervivencia de estas prótesis dento-implantosoportadas era alta, mayor que en el caso de las prótesis fijas sobre dientes e incluso algo superior que en el caso de los implantes.

Lindh et al. (2001) han visto, en un estudio llevado a cabo durante 2 años, que no se daba una tasa de fracasos superior a las restauraciones implantosoportadas. De hecho, la pérdida de hueso vertical alrededor del implante resultó ser menor en los casos de prótesis implantodentosoportadas, aunque en otros factores como la densidad de hueso, el tejido blando periférico o los índices de higiene y periodontal.

Olsson et al. (1995) han encontrado, en un estudio a 5 años de seguimiento, los siguientes datos:

- Supervivencia del implante: $88 \%$ ligeramente inferior a otros estudios, posiblemente debido a que situaron todos los implantes a nivel posteroinferior, de calidad ósea menor, donde la cresta alveolar es más estrecha o bien existe poco volumen óseo para alojar los implantes.

- Pérdida de soporte óseo: 0.1-0.3 mm durante el período de segumiento, de acuerdo con los resultados de otros autores. Sorprendentemente, hallaron que, tras los primeros 2 años de seguimiento no se dio ningún tipo de reabsorción, incluso se dio una ganancia en la altura marginal ósea.

- Tejidos marginales: no hallaron diferencias significativas con respecto a las prótesis implantosoportadas convencionales ni con respecto a los dientes contralaterales.

- Complicaciones técnicas: ninguna en particular.

\section{EVOLUCIÓN A LARGO PLAZO}

En lo que se refiere a la pérdida vertical de hueso, existen datos controvertidos. Como hemos visto en los estudios a corto plazo, los resultados parecen apuntar que existe una menor cantidad de reabsorción ósea en las 
prótesis implantodentosoportadas, pero en los estudios longitudinales, como el de Naert et al. se puede apreciar cómo la ferulización diente implante presenta una mayor actividad de reabsorción ósea que las prótesis implantosoportadas covencionales, que además es más patente en las conexiones rígidas, mientras que la conexión semirrígida no mostraba una diferencia significativa en cuanto a reabsorción ósea. El estudio de Naert et al. es bastante representativo, pues presenta una muestra de 133 pacientes y consiste en un estudio prospectivo a 15 años. En este estudio se puede apreciar cómo las conexiones no rígidas dienteimplante no presentan una diferencia significativa de pérdida ósea con respecto a las prótesis implantosoportadas convencionales (de unos $0.04 \mathrm{~mm}$ por año), mientras que en cuanto a las conexiones rígidas se producía una reabsorción mayor (0.09 mm por año).

\section{VENTAJAS}

- Psicológicas: Al emplear dientes naturales como pilares de prótesis, se reduce el número de implantes necesario y por tanto la complejidad y duración de la intervención quirúrgica.

- Terapia más conservadora: Del mismo modo, ante casos en los que vamos a colocar implantes y conectarlos mediante estructuras protésicas en los que es necesaria la extracción de dientes con soporte periodontal reducido, es posible plantearse la ferulización de dichos dientes a los implantes, como veremos en el capítulo de las indicaciones.

- Económicas: Igualmente, la menor colocación de implantes reduciría el coste de la intervención.

- Mantenimiento de la propiocepción: En el empleo de prótesis implantosoportadas, la pérdida del ligamento periodontal al no existir diente hace que el paciente no disponga plenamente de la capacidad propioceptiva, por lo que, manteniendo un diente como pilar natural en este tipo de prótesis es suficiente para que el paciente mantenga la propiocepción de la fuerza masticatoria que ejerce sobre la estructura protésica. Un estudio de Kay apoya esta hipótesis, pues ha visto un mayor índice de complicaciones en los casos de restauraciones mediante implantes que en los casos de restauración mediante dientes e implantes ferulizados: el paciente conserva el diente, con lo que obtiene una mejor y mayor información de la carga aplicada y puede evitar un estrés excesivo de la prótesis mediante un control más preciso de la fuerza de masticación ejercida. Cavicchia y Bravi (1999), según los datos arrojados por un estudio que realizaron evaluando las restauraciones protésicas implantológicas en comparación con la ferulización diente-implante, están de acuerdo con esta hipótesis, pues han observado un mayor índice de complicaciones relacionadas con el estrés en los casos de restauración mediante implantes únicamente.

- Mejor distribución de fuerzas: En la literatura, uno de los problemas que más están implicado en el origen del estrés en la estructura protésica es la falta de ajuste de la misma con los implantes. Está demostrado que esta falta de ajuste, inevitable en algunos casos, es más soportable en restauraciones apoyadas sobre dientes que en aquellas sobre implantes, y esto se debe a la presencia del ligamento periodontal. Si el implante constara de ligamento periodontal, o al menos dispusiera de algún elemento que le permitiera una cierta resiliencia, la falta de ajusta de la prótesis sería compensable desde este punto de vista, y esto es posible mediante la ferulización diente implante, en la que, además de dotar de cierta resiliencia a la estructura protésica implantosoportada merced a la parte dentosoportada, es posible añadir estructuras resilientes o intramóviles que reduzcan el estrés. Cavicchia y Bravi (1999) han visto que, si sólo se confía en el uso de estos elementos intramóviles para la reducción del estrés, el resultado es un fracaso, mientras que si se une el efecto amortiguador de estos componentes a la ferulización del rígido sistema implantológico al resiliente sistema periodontal de un diente natural, el resultado resulta ser el más efectivo y el que presenta menos riesgos. Esta mejor distribución de fuerzas también debería, en consecuencia, redundar en un mayor éxito a largo plazo, pues se daría un menor riesgo de pérdida ósea al existir una menor transmisión de fuerzas al lecho óseo circundante, pero no existen muchos estudios realizados a este respecto, y menos a largo plazo, por lo que esta aseveración se trata sólo de una hipótesis.

\section{DESVENTAJAS}

- Poca evidencia científica: Como hemos visto, existen pocos estudios al respecto de este tipo de prótesis, $y$, a pesar de que se han tenido en cuenta desde hace ya tiempo (Bränemark ya mencionó tácitamente su posibilidad) y que los estudios parecen ofrecer datos alentadores, la controversia sobre qué tipo de conexión debería realizarse sigue en pie, y así el profesional duda al plantear esta posibilidad ante un paciente, y más en la actualidad, ante la desafortunada aparición de una Odontología defensiva. Son necesarios más estudios, y mejor a largo plazo, y teniendo en cuenta el mayor número de variables 
posibles para determinar el diseño protésico óptimo y la viabilidad de la prótesis a largo plazo.

- Problemas en el diente pilar: Achacables a cualquier prótesis dentosoportada: la mayoría de las prótesis de este tipo se cementan sobre los dos pilares, con lo que tenemos una especie de irreversibilidad al cementarla, pues si el diente necesita posteriormente una endodoncia será necesario realizarla a través de la prótesis, debido a las características faraónicas de las estructuras protésicas cementadas a implantes. Puede ser necesario, de hecho, como han visto algunos autores, realizar la endodoncia antes de proceder a conectar la prótesis.

Por extensión en este aspecto, también hay que tener en cuenta las posibilidades de filtrado si las cargas que se ejercen sobre la prótesis son anómalas y provocan desplazamientos y déficits de sellado y ajuste marginal.

- Tallado de un diente natural: A pesar de la ventaja de ser más conservadora en líneas generales, estas prótesis implican el tallado de un diente natural al apoyarse sobre él, si bien se trata esta de una contraindicación justificada dada la necesidad de restauración en algunos pacientes.

\section{INDICACIONES}

- Socioeconomicas: En todo tratamiento Odontológico, es preciso tener en cuenta la realidad económica del paciente, que puede limitar nuestro tratamiento y nos puede obligar a buscar alternativas, como es el caso que nos ocupa. Una prótesis dentoimplantosoportada es más asequible, dentro de lo que cabe, que una prótesis implantosoportada convencional, y puede estar por ello indicada en pacientes con características socioeconómicas bajas.

- Alteraciones anatómicas: Casos de una edentación prolongada, con la reabsorción de la cresta residual y con una cercana presencia del seno maxilar en la arcada superior o del canal dentario en la inferior, en los que es difícil o imposible la colocación de implantes con el fin de restaurar estos sectores mediante una prótesis implantosoportada convencional, pueden ser una posible indicación para las prótesis implantodentosoportadas, en las que podemos colocar un menor número de implantes estratégicamente situados para ferulizarlos a los dientes remanentes.

- Número de implantes: Cuando existe un número insuficiente de implantes o dientes naturales que nos impidan la colocación de prótesis fijas convencionales.

- Distribución de pilares: Cuando la localización de los pilares no es favorable a lo largo de la arcada, sobre todo en lo que se refiere a los implantes que, en el momento de la planificación, debido a alteraciones anatómicas estructurales, se encuentran en una disposición poco alineada.

- Mejorar la versatilidad de la prótesis: Existen casos en los que es posible sospechar la pérdida de uno de los implantes si vamos a realizar una prótesis implantosoportada convencional. La pérdida de uno de los implantes acabaría con el futuro de la prótesis, pero podemos conseguir una mayor versatilidad de la prótesis para el futuro si ferulizamos el implante al diente, que actuaría como pilar auxiliar en caso de la pérdida de uno de los implantes.

- Dientes periodontales: Cabe plantearse el auxiliarse en un implante para, mediante la ferulización, conseguir solucionar un problema periodontal de movilidad en un diente con soporte periodontal reducido consecuencia posible de una periodontitis. Se ha visto a lo largo de la literatura (Kay, Naert et al...) que en estos casos el implante sufre las consecuencias de esta conexión, en la que las fuerzas oclusales impiden la restitución del diente periodontal y dificultan la osteointegración implantaria. Por ello sería considerado una contraindicación el empleo de este tipo de prótesis apoyándose en dientes periodontales. Sin embargo, según han visto algunos estudios (Chapman, van Rossen...) pudiera, según qué casos, tratarse de una contraindicación relativa si tenemos en cuenta el empleo de estructuras resilientes que absorban el estrés oclusal sobre el implante, como hemos visto con el sistema IMZ con lo que, en ciertos casos, podría indicarse la ferulización de un implante a un diente con una reducción no muy severa de su soporte periodontal con el fin de obtener su estabilización.

\section{CONCLUSIONES}

$1^{\circ}$ Existe aún poca literatura refiriéndose a las prótesis implantodentosoportadas, y, además, los estudios observados son en su mayoría a corto plazo, y no suelen tener en cuenta muchas variables, con lo que los resultados no se pueden aplicar con total seguridad, pues no se especifica el tipo de conexión realizada, el perfil del paciente... Son necesarios más estudios a largo plazo teniendo en cuenta todas las variables posibles.

$2^{\circ}$ Como hemos visto, parece ser que en general, las conexiones semirrígidas presentan, en un plazo más o 
menos variable, fenómenos de intrusión del diente natural, por lo que la tendencia viene a declinarse por las conexiones rígidas, si bien estas también presentan la desventaja de que la carga oclusal se concentra con más apetencia por el implante pilar, aumentando la reabsorción ósea y el riesgo de aflojamiento de tornillos y fractura de elementos. Se plantean numerosas posibilidades en la literatura para aumentar la resiliencia del implante pero, excepto el empleo de elementos intraimplantarios de resina compuesta, respecto al que existen pocos estudios, parece que su uso no está muy justificado por la escasa reducción de la transmisión del estrés al implante y el riesgo de fractura, y además, la reabsorción producida en las conexiones rígidas se estabiliza a los seis meses, y no parece suponer un problema a largo plazo, como han visto algunos estudios.

$3^{\circ}$ En resumen, parece que la mejor opción es, hoy por hoy, debido a la escasez de estudios adecuados el empleo de la prótesis implantosoportada convencional siempre que se pueda. Sin embargo, en algunos casos puede plantearse como opción válida si así simplificamos significativamente el tratamiento.

$4^{\circ}$ En un futuro no muy lejano es posible que, contando con evidencias científicas de la viabilidad de estos sistemas, se pueda plantear abiertamente la indicación de la prótesis implantodentosoportada en cualquier caso.

\section{SUIMIMARY}

A review about the tooth-implant splinting therapeutic option is presented. After a brief introduction, it begins with some biomechanical aspects. Then, some critical points about this kind of frames are shown in order to, afterwards, describe de different design that can be applied. A short and long-term evolution of these systems is also made and, finally, we can see some advantages, disadvantages and indications for the splinting option, to finish establishing some conclusions.

\section{KEYWORDS}

Tooth-implant connection, tooth-implant supported fixed prosthesis, splint.

\section{BIBLIOGRAFÍA}

1. Lill W., Matejka M., Rambousek K., Watzek G. The ability of currently available stress-breaking elements for osse- ointegrated implants to imitate natural tooth mobility. The International Journal of Oral \& Maxillofacial Implants 1988; 3: 281-6.

2. Brägger U., Aeschlimann S., Bürgin W., Hammerle C.H.F., Lang N.P. Biological and technical complications and failures with fixed partial dentures (FPD) on implants after four to five years of function. Clinical Oral Implant Research 2001; 12: 26-34.

3. Cavicchia F., Bravi F. Free-standing vs tooth-connected implant-supported fixed partial restorations: a comparative retrospective clinica study of the prosthetic results. The International Journal of Oral an Maxillofacial Implants 1999; 6: 711-7.

4. Naert I.E., Duyck J.A., Hosny M.M.F, Quirynen M., van Steenberghe D. Freestanding and tooth-implant connected prostheses in the treatment of partially edentulous patients. Clinical Oral Implants Research 2001; 12: 24551

5. Gross M., Laufer B.Z. Splinting osseointegrated implants and natural teeth in rehabilitation of partially edentulous patients. Part I: Laboratory and clinical studies. Journal of Oral Rehabilitation 1997; 24: 863-70.

6. Gross M., Laufer B.Z. Splinting osseointegrated implants and natural teeth in rehabilitation of partially edentulous patients. Part II: Principles and applications. Journal of Oral Rehabilitation 1998; 25: 69-80.

7. Cohen S.R., Orenstein J.H. The use of attachments in combination implant and natural-tooth fixed partial dentures: a technical report. The International Journal of Oral and Maxillofacial Implants 1994; 9: 230-4.

8. Olsson M., Gunne J., Astrand P., Borg K. Bridges supported by free-standing implants versus bridges supported by tooth and implant: A five-year prospective study. Clinical Oral Implant Research 1995; 6: 114-21.

9. Nishimura R.D., Ochiai K.T., Caputo A.A., Jeong C.M. Photoelastic stress analysis of load transfer to implants and natural teeth comparing rigid and semirigid conectors. The Journal of Prosthetic Dentistry 1999; 81: 696-703.

10. Lindh T., Back T., Nystrom E., Gunne J. Implant versus tooth-implant supported prostheses in the posterior maxilla: a 2-year report. Clinical Oral Implant Research 2001; 12: 441-9.

11. Mensor M.C., Ahlstrom R.H., Scheerer E.W. Compliant Keeper system replication of the periodontal ligament protective damping function for implants: Part I. The Journal of Prosthetic Dentistry 1998; 80: 565-9.

12. Mensor M.C., Ahlstrom R.H., Scheerer E.W. Compliant 
Keeper system replication of the periodontal ligament protective damping function for implants: Part II. The Journal of Prosthetic Dentistry 1999; 81: 404-10.

13. Giray B., Akça K., Iplikçioglu H., Akça E. Two-year followup of a patient with oligodontia treated with implant- and tooth-supported fixed partial dentures: A case report. The International Journal of Oral and Maxillofacial Implants 2003; 18: 905-11.

14. Romeo E., Lops D., Margutti E., Ghisolfi M., Chiapasco M., Vogel G. Long-term survival and success of oral implants in the treatment of full and partial arches: A 7year prospective study with the ITI Dental Implant System. The International Journal of Oral and Maxillofacial Implants 2004; 19: 247-59.

15. Wang T.M., Lee M.S, Kok S.H., Lin L.D. Intrusion and reversal of a free-standing natural tooth bounded by two implant-supported prostheses: A clinical report. The Journal of Prosthetic Dentistry 2004; 92: 418-22.

16. Hekimoglu C., Anil N., Cehreli M.C. Analysis of strain around endosseous dental implants opposing natural teeth or implants. The Journal of Prosthetic Dentistry 2004; 92: 441-6.

17. Kuzmanovic D.V., Payne A.G.T., Purton D.G. Distal implants to modify the Kennedy classification of a removable partial denture: A clinical report. The Journal of Prosthetic Dentistry 2004; 92: 8-11.

18. Mathews M.F., Breeding L.C., Dixon D.L., Aquilino S.A. The effect of connector design on cement retention in an implant and natural tooth-supported fixed partial denture. The Journal of Prosthetic Dentistry 1991; 65: 822-7.

19. Lindh T., Dahlgren S., Gunnarsson K., Josefsson T., Nilson H., Wilhelmsson P., Gunne J. Prótesis fijas soportadas por dientes e implantes: estudio multicéntrico retrospectivo. The International Journal of Prosthodontics 2001;14:3218.

20. Kay H.B. Free standing Versus Implant-ToothInterconnected Restorations: Understanding the Prosthodontic Perspective. The international Journal of Periodontal Restoration Dentistry 1993; 13: 47-69.

21. Ericsson I., Lekholm U., Branemark P.I., Lindhe J., Glantz P.O., Nyman S. A clinical evaluation of fixed-bridge restorations supported by the combination of teeth and osseointegrated titanium implants. The Journal of Clinical Periodontology 1986; 13:307-12.

22. Rieder C.A., Parel S.M., A Survey of Natural Tooth Abutment Intrusion With Implant-Connected Fixed Partial Dentures. The international Journal of Periodontal Restoration Dentistry 1993; 13: 335-47.
23. Rangert B., Gunne J., Glantz P.O., Svensson A. Vertical load distribution on a three-unit prosthesis supported by a natural tooth and a single Branemark implant. An in vivo study. Clinical Oral Implant Research 1995; 6: 40-6.

24. Palma V., Madrigal A., Pradíes G. Ferulizaciones dienteimplante. Revista internacional de Prótesis Estomatológica 2003; 5: 131-7.

25. Thams U., Rodríguez E. Prótesis telescópica galvanoformada soportada por implantes y dientes combinados. Revista Española Odontoestomatológica de impantes 2001; 9: 135-47.

26. Tangerud T., Gronningsaeter A.G., Taylor A. Fixed Partial Dentures Supported by Natural Teeth and Branemark System Implants: A 3-year Report. The International Journal of Oral and Maxillofacial Implants 2002; 17: 212-9.

27. Sheets C.G., Earthman J.C. Tooth intrusion in implantassisted protheses. The Journal of Prosthetic Dentistry 1997; 77: 39-45.

28. Schlumberger T.L., Bowley J.F., Maze G.I. Intrusion phenomenon in combination tooth-implant restorations: $A$ review of the literature. The Journal of Prosthetic Dentistry 1998; 80: 199-203.

29. Sada E., Manpaso C. Rehabilitación oral con prótesis fija, implantes y dientes naturales. Revista Española Odontoestomatológica de Implantes 2002; 10: 151-7.

30. Rodríguez A., Rodríguez F., Gago E. Rehabilitación mediante una prótesis implanto-dento-soportada con un interlock en su unión. Revista Española Odontoestomatológica de Implantes 1997; 5,4: 181-4.

31. Naert I.E., Duyck A.J., Hosny M.F., van Steenberghe D. Freestanding and tooth-implant connected prostheses in the treatment of partially endetulous patients. Part I: An up to 15-years clinical evaluation. Clinical Oral Implant Research 2001; 12: 237-44.

32. García L., Oesterle L. Natural Tooth Intrusion Phenomenon With Implants: A Survey. The International Journal of Oral and Maxillofacial Implants 1998; 13: 22731.

33. Fugazzotto P., Kirsch A., Ackermann K-L., Neuendorff G. Implant / Tooth Restorations Utilizing Screw-Fixed Attachments: A Survey of 3,096 Sites in Function for 3 to 14 Years. The International Journal of Oral and Maxillofacial Implants 1999; 14: 819-23.

34. Lindh T., Gunne G., Danielsson S. Rigid Connections Between Natural Teeth and Implants: A Technical Note. The International Journal of Oral and Maxillofacial Implants 1997; 12: 674-8. 
35. Clepper D.P., Ingber A., Jansen C.E., Stauts B. Should natural teeth and osseointegrated implants be used in combination to support a fixed prosthesis? The International Journal of Oral and Maxillofacial Implants 1997; 12: 855-9.

36. Menicucci G., Mossolov A., Mozzati M., Preti G. Toothimplant connection: some biomechanical aspects based on finite element analyses. Clinical Oral Implant Research 2002; 13: 334-341.

37. Weinberg L.A., Kruger B. Biomechanical considerations when combining tooth-supported and implant-supported prostheses. Oral Surgery Oral Medicine Oral Pathology 1994; 78: 22-7.

38. Uysal H., Iplikçioglu H., Avci M., Bilir O.G., Kural O. An Experimental Analysis of the Stresses on the Implant in an Implant-Tooth-Supported Prosthesis: A Technical Note. The International Journal of Oral and Maxillofacial Implants 1997; 12: 118-24.
39. Brunski JB. Biomechanical considerations in dental implant design. The International Journal of Oral Implantology 1988; 5:31-4.

40. McGlumphy EA, Campagni WV, Peterson LJ. A comparison of the stress transfer characteristics of a dental implant with a rigid or a resilient internal element. J Prosthet Dent. 1989 Nov; 62: 586-93.

41. Richter EJ. Basic biomechanics of dental implants in prosthetic dentistry. J Prosthet Dent. 1989 May; 61: 602-9.

42. Hutton JE, Heath MR, Chai JY, Harnett J, Jemt T, Johns RB, McKenna S, McNamara DC, van Steenberghe D, Taylor R, et al. Factors related to success and failure rates at 3-year follow-up in a multicenter study of overdentures supported by Branemark implants. Int J Oral Maxillofac Implants. 1995 Jan; 10: 33-42. 